\title{
The influence of two-dimensional stimulus properties in a reconstruction task with minimal information stimuli
}

\author{
STEFAAN TIBAU, BERT WILLEMS and JOHAN WAGEMANS* \\ Department of Psychology, University of Leuven, Tiensestraat 102, B-3000 Leuven, Belgium
}

Received 2 February 2000; revised 17 April 2000; accepted 28 April 2000

\begin{abstract}
Effects of viewpoint that are typically found in shape recognition tasks have been interpreted in the past as strong evidence against approaches based on the use of geometric invariants by the human visual system. At first sight, the use of such invariants would indeed predict shape recognition to be viewpoint independent. It has been argued before, however, that the visual estimation of invariants need not itself be invariant. Changes of viewpoint introduce changes in $2 \mathrm{D}$ stimulus features. The latter may make the estimation of invariants by the visual system more or less difficult. The present study explores how such 2D features influence estimation distributions in a reconstruction paradigm. Subjects were shown four coplanar dots. They were asked to estimate the position of one of the dots relative to the three others on the basis of a slanted version of the configuration. An estimation distribution was obtained for every position of the dot to be estimated. Biases in these distributions indicated that subjects performed the task by using $2 \mathrm{D}$ reference axes that were unambiguously identifiable in both the images of the dot pattern.
\end{abstract}

Keywords: Affine transformation; collinearity; parallelism; pattern matching; qualitative features; shape perception; viewpoint invariance.

\section{INTRODUCTION}

When we look at a figure from two different points of view, we can often see that it is the same figure, despite the differences in the projections on our retina that are due to the change in viewpoint. Gibson (1979) argued that this is possible through the use of invariants. Invariants are geometrical properties of the retinal projections of shape that remain constant under changes in orientation of the original threedimensional (3D) shape (see Cutting, 1983, 1986; Forsyth et al., 1991; Mundy and Zisserman, 1992; Van Gool et al., 1994a, for discussions of specific geometric invariants).

The use of invariants by the human visual system has been investigated in a range of experimental paradigms from rigidity detection with moving stimuli (Cutting,

\footnotetext{
*To whom correspondence should be addressed. E-mail: johan.wagemans@psy.kuleuven.ac.be
} 
1986; Simpson, 1983), and detection of equivalence of pairs of shapes that differ by some transformation (e.g. Euclidean, similarity, affine, projective) (Niall and Macnamara, 1990; Pizlo, 1994; Wagemans et al., 1997, 2000), to reconstruction of frontoparallel shapes from an in-depth version of the same shape (Niall and Macnamara, 1989, 1990; Niall, 1997). The evidence with regard to the actual use of invariants remains mixed (see Note 1 at end of paper). However, from this literature it becomes clear that, if the visual system uses invariants, their estimation in the image is itself not invariant over different viewing positions.

This is an important point because the heaviest critique on the invariants-based approach of shape recognition is based on evidence that human performance in shape recognition task is not completely independent of the orientation of the shape that is to be recognized. It has been shown that the time and accuracy to recognize a shape that is oriented differently than a previously learned version of the same shape tend to vary with the difference in orientation between the two (e.g. Jolicoeur, 1985; Jolicoeur and Landau, 1984; see Lawson, 1999, for a review). These results have typically been interpreted as evidence for the presence of a mental rotation mechanism (e.g. Ullman, 1989), which is supposed to mentally undo the spatial transformation between the learned version and the version that is currently seen. At first sight, one would indeed not expect recognition of shapes to depend on the orientation of the shape if recognition were based on invariants.

However, Wagemans et al. (1996) suggested an alternative explanation, which holds that maybe the visual estimation of invariants in itself is not invariant. They argued that the estimation of invariants by the visual system is influenced by twodimensional (2D) properties. Since orientation changes induce changes in these properties, they might also make the estimation of the invariants more or less difficult. In their experiment, subjects explicitly estimated the affine invariant coordinates of a dot relative to three other dots. Koenderink and Van Doorn (1991) have shown that three non-collinear points in an orthographic projection of a planar shape define an affine reference frame. These anchor points form the unit vectors of the frame. The location of every other point relative to the three fixed points can be expressed in terms of coordinates on the unit vectors. These coordinates are invariant under affine transformation (see Fig. 1). Wagemans et al. (1996) showed their subjects four dots and asked them to express the location of one of the four dots as the affine coordinates relative to the other three. Two-dimensional properties such as the angle formed by the basis vectors of the affine reference frame and the aspect ratio of these basis vectors had a profound influence on the performance in this task.

In the present paper we want to further investigate how such properties influence perception of $2 \mathrm{D}$ dot configurations that are slanted in depth, by looking at biases in the estimation distributions obtained by means of a reconstruction task. Subjects were shown two dot patterns consisting of four dots each: one that is slanted in depth and a frontoparallel version of the first one. In the latter pattern, three dots are fixed while the fourth dot had to be positioned by the subjects, such that the 

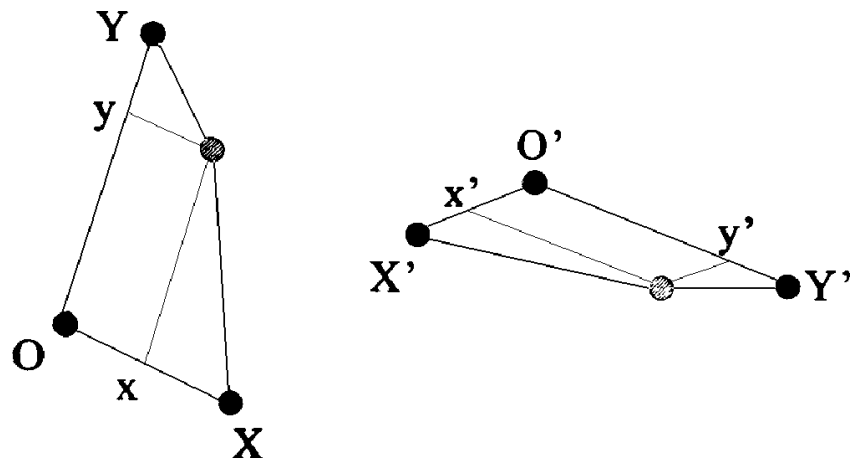

Figure 1. An example of two affine equivalent four dot configurations. The affine coordinates of the shaded dot relative to the reference frame defined by the three solid dots are equal to each other in both of the images (i.e. 0.50, 0.75).

slanted pattern was reconstructed. Doing this several times at a fixed position yields an estimation distribution.

Such a distribution may provide information about the information in the stimulus that subjects used while they were doing the task. For example, Phillips et al. (1997) used a similar technique to investigate dot localization on curved surfaces. The pattern of mean errors and variances allowed them to draw conclusions about the information that the subjects used (namely, curvature minima and maxima). We will also look at the form of the error distributions. This will be done by fitting covariance ellipses to the estimations and then seeing whether they are significantly elongated in some conditions. Elongation is an indication that the estimations are somehow more restricted in one direction than in the other. This suggests in turn the presence of attributes that are relevant for doing this task.

\section{METHODS}

\subsection{Subjects}

Ten subjects from the Laboratory of Experimental Psychology at the University of Leuven participated in the experiment. Seven of the 10 were naive about the purpose of the experiment. The three others were the authors. All subjects had normal or corrected-to-normal vision.

\subsection{Apparatus}

The experiment was run on an IBM compatible PC with a Pentium processor, with a clock speed of $120 \mathrm{MHz}$. We used an SVGA monitor with a temporal resolution of $70 \mathrm{~Hz}$ and a spatial resolution of $800 \times 600$ to present the stimuli. Stimuli were viewed in a completely darkened room, through a tunnel that was clothed on the inside with a black light absorbing material. The tunnel's length was approximately $60 \mathrm{~cm}$. 


\subsection{Stimuli and task}

Stimuli consisted of four coplanar dots that were slanted in depth and orthographically projected onto the computer screen. This is what we call the 'model pattern'. Subjects could switch to a frontoparallel version of the model pattern, the 'test pattern', by pressing the left mouse button. In the test pattern the fourth dot was not fixed, but could be moved by the mouse. The task of the subjects was to position the fourth point in the test pattern from what they had seen in the model pattern. They did this by moving the cursor and verifying by alternating between the model and the test pattern until they were satisfied with the setting. They entered the value by clicking on the right mouse button.

To avoid apparent motion, the model and test pattern were separated in time and space. Between the appearance of the model and test pattern, there was a brief period of $750 \mathrm{~ms}$ during which the screen was black. The patterns were also separated spatially: they always appeared in diagonally opposite quadrants of the screen.

The four dot patterns were constructed as follows. We started from seven configurations that only differed from each other in the position of the fourth dot relative to the other three. In what follows, we will express this position relative to the affine coordinate frame formed by the other three dots (see Introduction and Fig. 1). There were nine possible positions in the experiment: $(0.25,0.25),(0.25$, $0.75),(0.25,1.25),(0.75,0.25),(0.75,0.75),(0.75,1.25),(1.25,0.25),(1.25,0.75)$ and $(1.25,1.25)$. These were the original configurations from which we constructed both the model and test pattern (see Fig. 2).

First, the dots were oriented upright, such that the symmetry axis of the affine reference frame coincided with the vertical. This was done in order to keep the basis vectors equally long, even after the pattern had been slanted. Next, the dots were slanted (rotated along a horizontal axis) 0, 55 or 71 degrees. Finally, a rotation around the line of sight (tilt) was applied. This pattern was projected

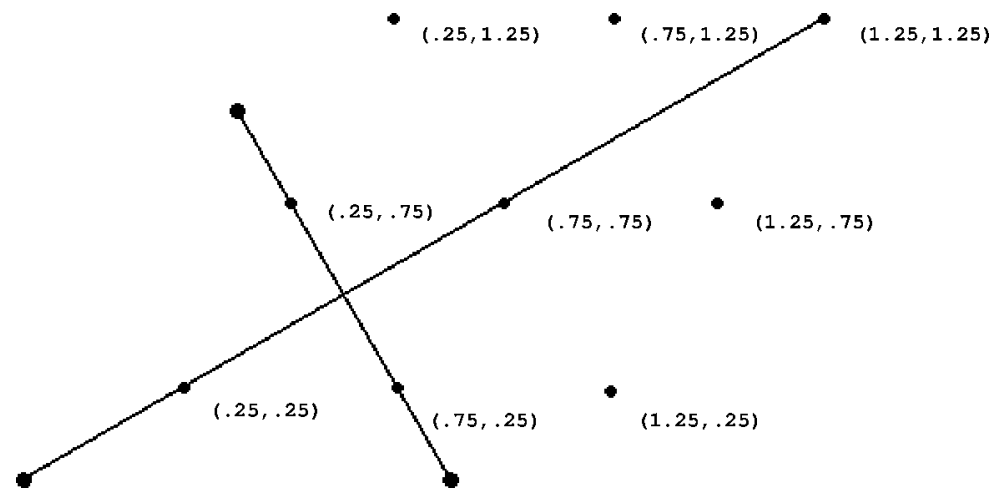

Figure 2. The different positions of the fourth dot that the subjects had to estimate during the experiment. The two lines indicate what we call the symmetry axis and the collinearity axis in the text. 
orthographically onto the computer screen. The test pattern was not slanted but had the same tilt value as the model pattern.

The length of the basis vectors was 120 pixels (4.8 visual deg). The smallest distance of the fourth dot to the origin of the affine reference frame was 52 pixels (2.1 visual deg). This was the case in the conditions where the fourth dot was at the position $(0.25,0.25)$. The largest distance between the fourth dot and the origin occurred in the $(1.25,1.25)$ conditions. This distance was 259 pixels $(10.5$ visual degrees). In all of the other conditions the distance from the fourth dot to the origin was between these values.

In order to make it easier for the subjects to solve the correspondence problem (i.e. the problem of which dot in the model pattern corresponds to which dot in the test pattern), the origin of the affine reference frame was red, the dots defining the basis vectors were green. The fourth dot was white. This configuration was shown on a black background.

\subsection{Procedure}

There were 27 experimental conditions (three levels of slant and nine levels of position). Each of these conditions was repeated 20 times yielding 540 trials per subject. All of the subjects went through seven sessions. The first five sessions consisted of 72 trials each. During these sessions, only slanted model patterns were shown (only slant values of 55 and 71 degrees occurred). The stimuli were shown in random order throughout the five sessions. Then, two additional sessions with each 90 trials followed, in which the model patterns all had a slant of zero. During these sessions the task was reduced to a copy task instead of a reconstruction task. They were used as control conditions.

Prior to the actual experiment, subjects went through a preparatory session. They were first given instructions. The task was explained and a demonstration was given of how one could switch back between the model and test pattern, position the fourth dot, and register an answer. Then the subjects did a series of practice trials in which each of the slanted model patterns (18 stimuli) appeared once. We did this to allow the subjects to familiarize themselves with the stimuli. No feedback was given, unless it was apparent that the subject had not properly understood the instructions (e.g. when he/she placed the fourth dot outside of the reference frame).

\section{RESULTS}

\subsection{Dependent variables}

There were 20 trials per condition per subject. This yielded a cloud of 20 dots per condition that represented the subject's estimations. A covariance ellipse, centered on the 2D median of the estimations, was fitted through each of these clouds (see te Pas et al., 1998, for a similar technique). The mathematical details of these calculations are given in Appendix A. 

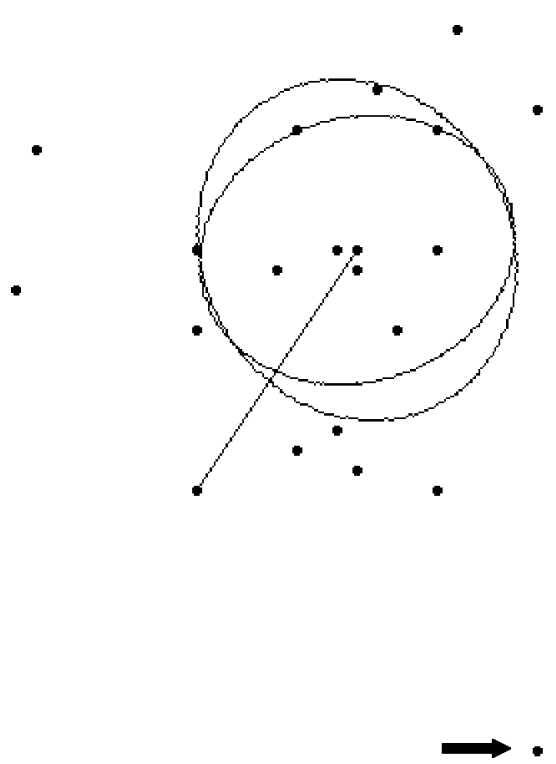

Figure 3. The calculation of the covariance ellipses. The larger ellipse takes all of the 20 data points into account. This ellipse is used to decide which data points are considered to be outliers (indicated by an arrow). After the outliers are removed, a second covariance ellipse is fitted to the data. This is the smaller ellipse.

Covariance ellipses were calculated twice. After the first covariance ellipse was calculated, outliers were removed. Then the covariance ellipse was calculated again on the basis of the remaining data points (see Fig. 3). Data points were considered outliers if their distance to the midpoint of the ellipse was at least three times the distance between the midpoint of the ellipse and the ellipse contour along the direction of the data point. Most of the time, there were no outliers. The maximum number of outliers that had to be removed this way was two out of 20 .

The parameters of the covariance ellipses were then averaged over subjects in every condition. This yielded one covariance ellipse in each of the conditions (see Fig. 4). Several measures were derived from them that were subsequently used in statistical analyses: (1) the distance between the 2D median of the estimations and the veridical position of the fourth dot, (2) the size of the covariance ellipse, and (3) its orientation.

\subsection{Deviation from the veridical position of the fourth dot}

This dependent variable was calculated as the distance between the 2D median and the veridical position of the fourth dot. An analysis of variance (ANOVA) revealed that Slant and Position, as well as their interaction, were significant. The $F$-values are, respectively: $[F(2,18)=56.51 ; p<0.0001],[F(8,72)=6.83 ; p<0.0001]$ and $[F(16,144)=2.78 ; p<0.001]$. From Fig. 5 it can be seen that the average absolute errors were small. The largest absolute error that occurs was somewhat 


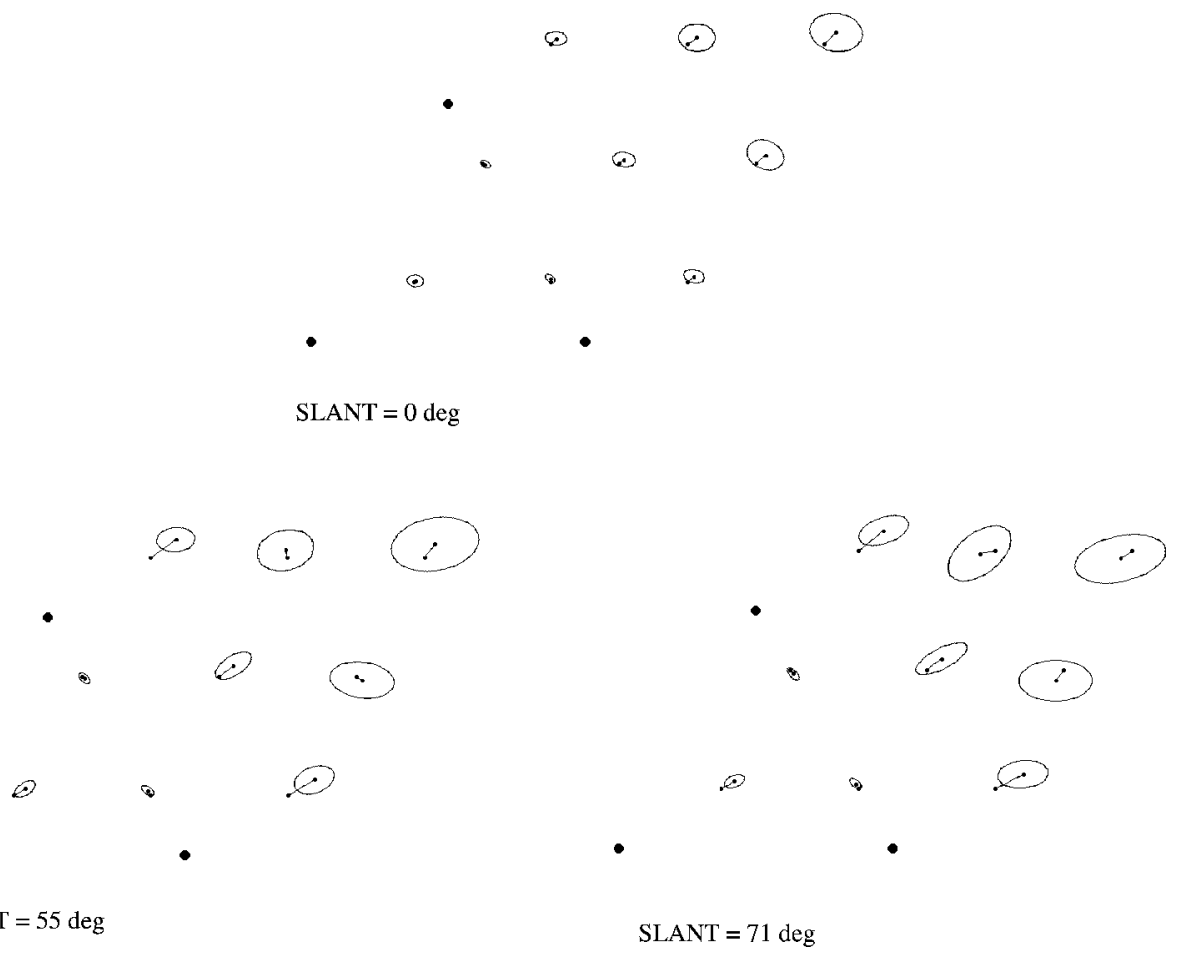

Figure 4. The average covariance ellipses that were obtained in all of the experimental conditions.

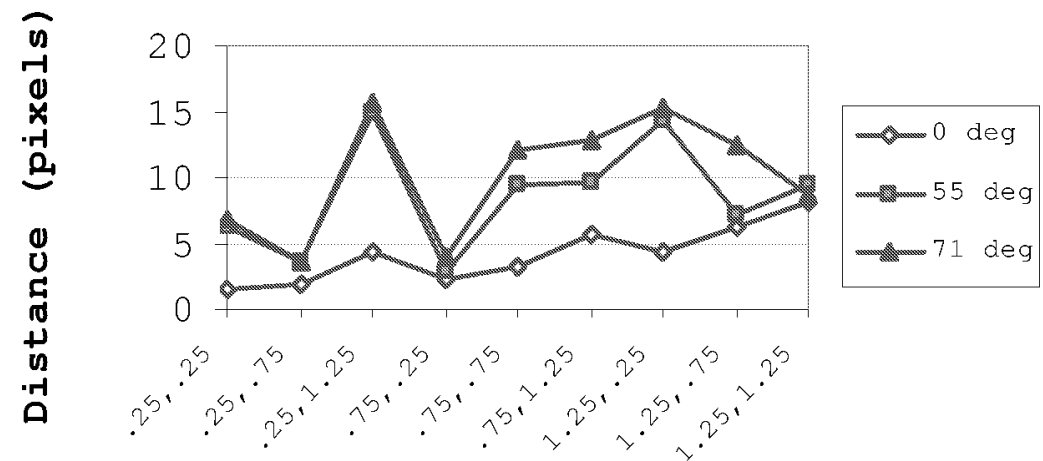

Position

Figure 5. The effect of Slant and Position on the distance between the veridical position of the point to be estimated and the 2D median of the covariance ellipses.

smaller than 16 pixels (1.02 visual degrees). This is the case in the 71 degrees slant condition at position $(0.25,1.25)$. If we express this error in terms of affine coordinates it amounts to $4.9 \%$ along the $X$-axis and $8.6 \%$ along the $Y$-axis of the affine reference frame. This is comparable to the sizes of the average errors 


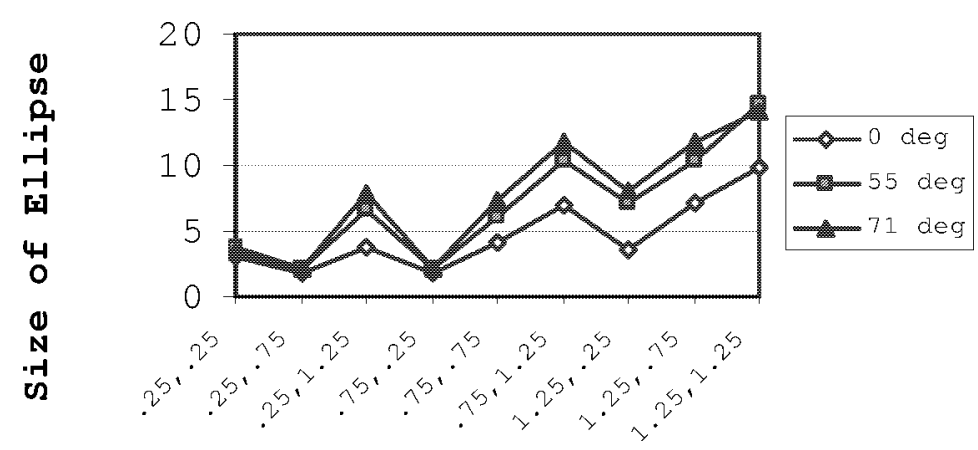

Position

Figure 6. The effect of Slant and Position on the size of the covariance ellipses.

found by Wagemans et al. (1996), although they did not use a reproduction task (see Introduction): $5.8 \%$ on the $X$-axis and $7.5 \%$ on the $Y$-axis.

A Tukey a posteriori analysis revealed that the average deviation from the veridical position did not depend on the slant at the positions $(0.25,0.25),(0.25$, $0.75),(0.75,0.25),(1.25,0.75),(0.75,1.25)$ (see Note 2$)$ and $(1.25,1.25)$. At $(0.25,1.25)$ and $(1.25,0.25)$ both the 55 and 71 degrees slant conditions differed significantly from the zero slant conditions. Finally, at $(0.75,0.75)$ there was a significant difference between the zero and the 71 degrees slant condition.

\subsection{Size of the covariance ellipse}

We calculated the size of the covariance ellipse dividing both the axes of the ellipse by two and then taking the square root of their product. This quantity can be used as a measure for the spread of the estimations. Another analysis of variance (ANOVA) yielded significant effects for Slant $[F(2,18)=74.51 ; p<0.0001]$, Position $[F(8,72)=146.01 ; p<0.0001]$ and the interaction between these factors $[F(16,144)=9.15 ; p<0.0001]$.

Figure 6 shows how the size of the covariance ellipses varies with Slant and Position. Tukey a posteriori analyses yielded significant effects between the zero slant conditions on the one hand and 55 and 71 degrees slant conditions on the other hand at all positions, except for $(0.25,0.25),(0.25,0.75)$ and $(0.75,0.25)$. These are the positions that are closest to the reference points. They are collinear with the two reference points that form the basis of the affine reference frame. Since uncertainty exists only in the direction perpendicular to the direction of the collinearity (the direction of slant), the position is completely determined by the $2 \mathrm{D}$ information in the stimulus at these positions.

\subsection{Orientation of the covariance ellipses}

First, in each condition we tested whether the covariance ellipses had a preferred orientation versus whether their orientations were uniformly distributed between 0 


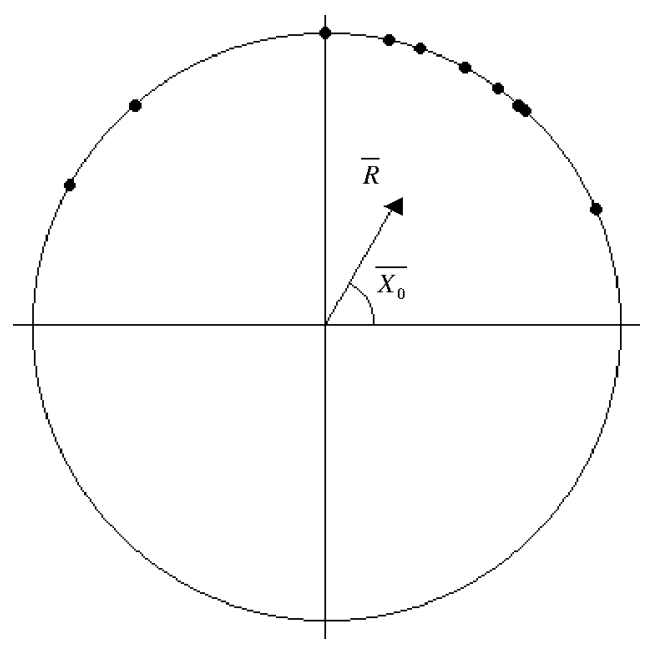

Figure 7. A sample of orientation data can be described by its mean orientation, $\bar{X}_{0}$, and its uniformity, $\bar{R}$. This can be represented visually by the orientation and length of a vector on a unit circle respectively.

and $\pi$ radians (see Note 3 ). Then, if there was a preferred orientation, we tested whether it was along the symmetry or collinearity axis. Mardia (1972) describes the test statistics and their distributions that are needed.

Axial data can be thought of as points lying on half a unit circle, thus as vectors with unit length and a given orientation (see Fig. 7). From these vectors, a resultant vector can be calculated that can be described by its orientation, $\bar{X}_{0}$, and length, $\bar{R}$. The value $\bar{R}$ can then be used as a test statistic to test the hypothesis that the vectors are distributed uniformly along the interval $[0, \pi]$. The higher the value of this measure, the more likely it is that there is a preferred orientation in the data. If there is a preferred orientation, $\bar{X}_{0}$, we can check whether the hypothesis that it is equal to a given theoretical orientation, $\mu_{0}$, can be rejected. Further details of the statistical procedures that we used here are given in Appendix B.

The results of the analysis we did on the orientations of the covariance ellipses are shown in Fig. 8A, B and C for each of the respective slant values. Each of the unit circles represents a condition of our experimental design (27 conditions). The dots on the unit circles represent the orientations of the covariance ellipses of the ten subjects. The line that is drawn from the center of the unit circles is the resultant vector of these orientations. Its orientation is the mean orientation, $\bar{X}_{0}$, and its length is $\bar{R}$. The values of these parameters are shown beneath their corresponding unit circles. Data points that were not included in the analyses are represented as open circles on the unit circle. It is also indicated in which conditions $\bar{R}$ is significant at the $\alpha=0.05 / 27$ level and if so, whether the orientation of the symmetry or collinearity axis is in the $95 \%$ confidence interval (see Note 4 ) about the mean orientation. This is indicated by means of a line below the value of the test statistic. Dotted lines refer to marginally significant effects. 


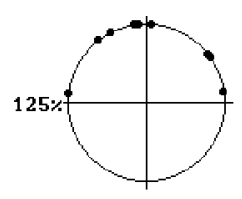

$\mathrm{R}=0.22 \quad \mathrm{x}=90$

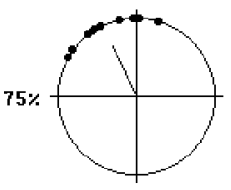

$R=0.69 \quad x=114$

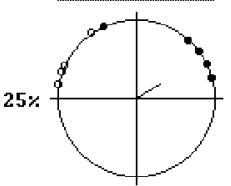

$R=0.59 x=28$

$25 x$

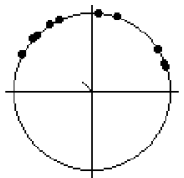

$R=0.15 \quad X=132$

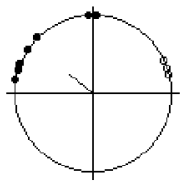

$R=0.51 \quad X=142$

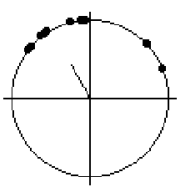

$R=0.48 x=118$

$75 \%$

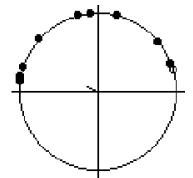

$R=0.14 x=161$

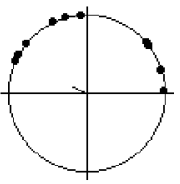

$\mathrm{R}=0.19 \quad X=154$

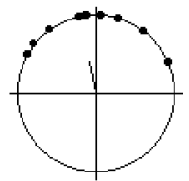

$\mathrm{R}=0.40 \quad \mathrm{x}=99$ $125 \%$

Slant $=0 \mathrm{deg}$

(A)
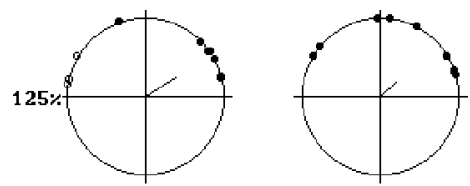

$\mathbf{f}=0.26 \quad x=40$
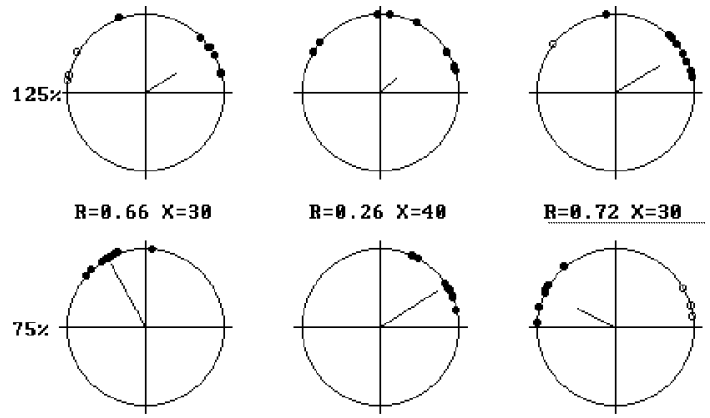

$F=0.72 x=39$

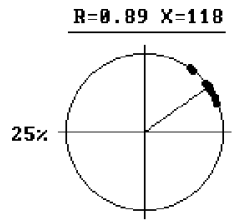

$\mathrm{H}=0.85 \quad \mathrm{X}=32$

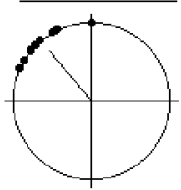

$\underline{H=0.81 \quad x=130}$

$75 \%$

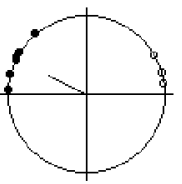

$R=0.88 \quad x=154$

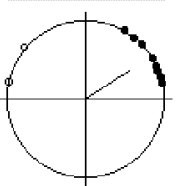

$\mathrm{B}=0.84 \quad \mathrm{x}=32$ $125 \%$

Slant $=55 \mathrm{deg}$

(B)

Figure 8. The orientation of the covariance ellipses in every condition. Every dot on a unit circle refers to the orientation of the covariance ellipse of one subject in that condition. Open circles indicate outliers. The orientation of the line that extends from the center of the unit circle indicates the mean orientation, $\bar{X}_{0}$, of the data in that condition. Its length indicates the uniformity of the sample. 


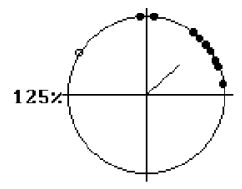

$\mathrm{H}=0.63 \quad \mathrm{x}=42$
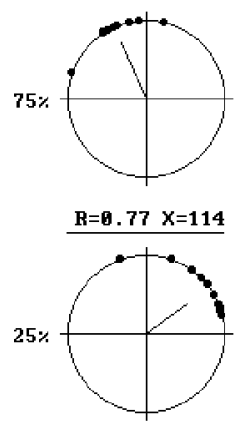

$\mathrm{P}=0.65 \quad x=36$

$25 \%$

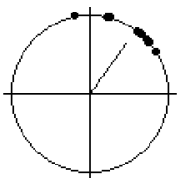

$\mathrm{H}=\mathbf{0 . 7 8} \mathrm{X}=54$

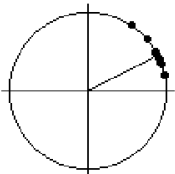

$\mathbf{R}=0.92 \quad x=27$

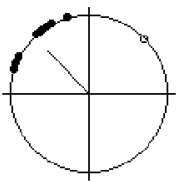

$\underline{B=0.02 \quad x=133}$

$75 \%$

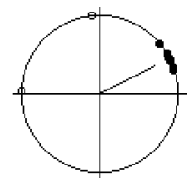

$\underline{B=0.97 \quad X=26}$

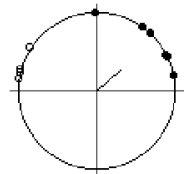

$P=0.67 \quad x=39$

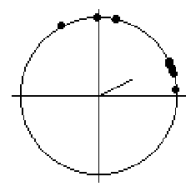

$\mathrm{R}=0.47 \quad \mathrm{X}=24$

$125 \%$

Slant $=71 \mathrm{deg}$

(C)

Figure 8. (Continued).

When there is no difference in slant between the model and the test pattern ( slant $=0 \mathrm{deg}$ ), the hypothesis that the orientations of the covariance ellipses are uniformly distributed on the unit circle cannot be rejected at any of the positions of the fourth dot, except for $(0.25,0.75)$ which has a marginally significant nonuniform distribution along the collinearity axis. This axis is within the 95\% confidence interval around the mean orientation in this condition.

When the slant is $55 \mathrm{deg}$, there is a preferred orientation on the symmetry axis at the positions $(0.25,0.25)$ and $(0.75,0.75)$ and on the collinearity axis at the positions $(0.25,0.75)$ and $(0.75,0.25)$. In all of these respective conditions, the symmetry and collinearity axes were in the $99 \%$ confidence interval about the mean orientation. Positions $(1.25,0.25),(1.25,0.75)$ and $(1.25,1.25)$ showed marginally significant orientations. The symmetry axis was in the confidence interval at (1.25, $0.25)$ and at $(1.25,1.25)$. None of the axes was in the interval at $(1.25,0.75)$.

At the slant level of $71 \mathrm{deg}$, there are preferred orientations at the positions $(0.75$, $0.75),(1.25,1.25),(0.25,0.75)$ and $(0.75,0.25)$. The first two positions are on the symmetry axis while the latter two are on the collinearity axis. Again, the proper axes are in the confidence interval at all of these positions. Position $(0.75,1.25)$ also had a preferred orientation, but none of the reference axes was in the confidence interval about its mean orientation.

Finally, we did a test to check whether the expected pattern of preferred orientations and their coincidences with the reference axes was statistically the same as the one we observed. To perform such a test, we have to start by constructing two two-way frequency tables, one for the expected pattern of orientations and one for 
Table 1.

Results of the $\chi^{2}$-analysis on the pattern of significances

\begin{tabular}{lcc}
\hline \multicolumn{2}{c}{ S } & NS \\
\hline \multicolumn{2}{l}{ Observed pattern of significance } & \\
$\mathrm{S}$ & $8 / 27$ & $2 / 27$ \\
$\mathrm{NS}$ & $4 / 27$ & $13 / 27$ \\
\multicolumn{2}{l}{ Expected pattern of significance } & \\
$\mathrm{S}$ & $10 / 27$ & 0 \\
$\mathrm{NS}$ & 0 & $17 / 27$ \\
\hline
\end{tabular}

The observed pattern of significance was obtained as follows:

(1) If a condition had a significant $\bar{R}$-value and if the right axis (i.e. the one in which the fourth dot was lying in this condition) was in the $95 \%$ confidence interval about the mean orientation, the condition was categorized as S/S (upper left quadrant).

(2) If a condition was expected to have a significant orientation along one of the axes but it did not meet the requirements described in (1) it was categorized as S/NS (upper right quadrant).

(3) If a condition was expected not to be significant but it was (fully or marginally), it was categorized as NS/S (lower left quadrant).

(4) The NS/NS category (lower right quadrant) contained the conditions that were expected not to have any significant orientation and that did not.

the observed pattern. Table 1 shows these frequencies. We expected the conditions $(0.25,0.25),(0.75,0.75),(1.25,1.25),(0.25,0.75)$ and $(0.75,0.25)$ to be significantly elongated along the axis (symmetry or collinearity) on which they were lying, when the slant was not equal to zero (55 deg and $71 \mathrm{deg}$ ). That means there is a preferred direction and this direction is along the axis on which the point is lying. All the other conditions were expected to have no preferred direction. Thus, a proportion of $8 / 27$ conditions were expected to yield significant preferred directions that did not deviate statistically in orientation from the axis on which they were lying, while the rest of the conditions $(17 / 27)$ were expected not to produce any significant effects. However, the observed proportions were slightly different (see Table 1$)$. The conditions $(1.25,1.25)$ at $55 \mathrm{deg}$ slant and $(0.25,0.25)$ at $71 \mathrm{deg}$ slant had only a marginally significant preferred direction and were subsequently placed in the upper right quadrant of the frequency table. There were also conditions that we expected not to show any preferred orientations but that nevertheless did (marginally). These were $(0.25,0.75)$ at 0 deg slant, $(1.25,0.25)$ and $(1.25$, $0.75)$ at $55 \mathrm{deg}$ slant and finally $(0.75,1.25)$ at $71 \mathrm{deg}$ slant. A chi-square test showed that these two patterns of frequencies did not deviate statistically from each other $\left(\chi^{2}=1.341 ; p=0.719\right)$. Hence, the observed pattern of significances does not deviate from what could be expected theoretically.

\subsection{Conclusion}

The results of the experiments show that, although the precision of the estimates depends on both Slant and Position, the deviation of the median estimated point from the veridical point in the different conditions is small, even when the model 
pattern is slanted. The same goes for the spread of the estimation errors. Thus, it can be concluded that performance is good on average. However, the fact that these dependent variables differ between conditions shows that different information is available for solving the task. The orientations of the covariance ellipses along the symmetry and collinearity axes suggest that subjects used these axes as qualitative reference axes that can be identified unambiguously in both the model and test pattern. They carve up the space defined by the three anchor dots, so as to delineate small regions within which estimations of the position of the fourth dot can be made. This way a reasonable average precision can be attained without any need for indepth interpretations of the stimuli.

\section{GENERAL DISCUSSION}

To complete the present task, subjects seem to use lines for which correspondence in the two images of the stimulus can be established. These are defined by unambiguously identifiable points. In principle, any feature that can be identified in $2 \mathrm{D}$ in both of the views of a shape can serve the same reference function for other positions (off the reference axes). They allow for a not so precise retrieval of these positions that is nevertheless less effortful than a more complex calculation of invariants that would yield precise estimates of position (see also Wagemans et al., 2000).

The importance of such stimulus features has been pointed out in the past. Foster (1979, 1980) proposed that visual patterns are represented internally by means of discrete structural descriptions of the stimulus that are associated with it in a probabilistic way. The building blocks of such descriptions are supposed to be local pattern features such as fixed lines, curves, vertices, collinearities, etc. and the relationships between them. He also suggested that such features can be regarded as fuzzy properties (Ferraro and Foster, 1994; Foster, 1977).

Based on these ideas, Kukkonen et al. (1996) formulated a model for the detection of affine shape equivalence on the basis of qualitative features such as collinearity, parallellism, convexity / concavity. The model treats these properties as continuous properties in the sense that the thresholds for discriminating between two planar shapes in 3D, vary with the extent to which the aforementioned properties are present in the stimulus. Performance is good if the stimuli contain collinear or nearly collinear dots. The less collinearity the stimulus contains, the more difficult the discrimination becomes. The same reasoning could be applied to our data. The position of the dots to be estimated can be more/less collinear with the collinearity axis than with the symmetry axis. They form a kind of fuzzy border on which the estimations can be based.

In the present experiment we used reference lines as such features, but these are only one example of what qualitative reference features may be. Van Gool et al. (1994b, 1995) proposed a complete hierarchy of features such as points, lines, lines of fixed points (e.g. collinear points that can be matched across views), and pencils 
of lines. Which features one is dealing with depends on the specific transformation of the 3D shape. But, given a certain type of transformation, one can unambiguously identify some of these features over different views of the shape. A range of useful predictions can be made from such a theory, some of which are currently being tested in our laboratory. The finding that the symmetry axis and the collinearity axis are used in the present experiment is a first indication that such a mathematical framework has some relevance to human vision.

We consider the present study as an exploratory analysis into the biases that emerge in reconstruction tasks such as the one we used in this experiment. This yielded some insight in the reference features that our subjects used during the task. In the long run it should be possible to dissociate between effects in shape recognition tasks that are due to orientation and position differences on the one hand, and effects that are due to changes in 2D stimulus features that occur as a consequence of the orientation changes. Effects of these features should be filtered out of the performance. Only then does it become possible to measure viewpoint (in)dependency.

\section{NOTES}

1. One aspect of this issue is theoretical: How should experiments be designed that address the role of invariants in human vision? For example, Niall (2000) has recently criticized some of our work (Wagemans et al., 1997) on the basis of a different view on the role of invariants and the way one should test it. We are currently preparing a reply to his critique. This theoretical discussion is not directly relevant to the present research.

2. At this position there was a marginally significant difference between the zero and the 71 degrees slant conditions $(p=0.06)$.

3. The interval is restricted to $[0, \pi]$ because we are dealing with axial data. Ellipses do not have a direction, only an orientation.

4. We did not correct the size of the confidence interval for the number of simultaneous tests for two reasons: (1) it was not clear a priori how many simultaneous tests we would have to conduct and (2) because in this case the tests are stronger when the confidence is small.

5. The three axes did not necessarily cross in one point. They much rather formed a very small triangle (often invisible as in Fig. A1). The actual coordinates of the 2D median were taken to be the midpoint of this triangle.

\section{Acknowledgement}

This research was supported by a research grant from the Regional Impulse Program for the Humanities (CAW 96/07) and from the Research Program of the Fund for Scientific Research-Flanders (FWO G.0130.98) to JW. We would like to thank Mario Pandelaere for useful advice about statistical analyses and James Todd for 
helpful discussions. We also thank two anonymous reviewers for their remarks, which have improved the quality of the presentation.

\section{REFERENCES}

Cutting, J. E. (1983). Four assumptions about invariance in perception, J. Exper. Psychol.: Human Perception and Performance 9, 310-317.

Cutting, J. E. (1986). Perception with an Eye for Motion. MIT Press, Cambridge, MA.

Ferraro, M. and Foster, D. H. (1994). Elements of a fuzzy geometry for visual space, in: Shape in Picture, Toet, A., Foster, D. H., Heijmans, H. and Meer, P. (Eds), pp. 333-342. Springer-Verlag, Berlin.

Forsyth, D., Mundy, J. L., Zisserman, A., Coelho, C., Heller, A. and Rothwell, C. (1991). Invariant descriptors for 3D object recognition and pose, IEEE Trans. Pattern Analysis and Machine Intelligence 13, 971-991.

Foster, D. H. (1977). Optica Acta 24, 147- 157.

Foster, D. H. (1979). Discrete internal pattern representations and visual detection of small changes in pattern change, Perception and Psychophysics 26, 459-468.

Foster, D. H. (1980). A description of discrete internal representation schemes for visual pattern discrimination, Biological Cybernetics 38, 151-157.

Gibson, J. J. (1979). The Ecological Approach to Visual Perception. Houghton Mifflin, Boston, MA.

Jolicoeur, P. (1985). The time to name disoriented natural objects, Memory and Cognition 13, $289-303$.

Jolicoeur, P. and Landau, M. J. (1984). Effect of orientation on the identification of simple visual patterns, Canad. J. Psychol. 38, 80-93.

Koenderink, J. J. and van Doorn, A. J. (1991). Affine structure from motion, J. Opt. Soc. Amer. 8, $377-385$.

Kukkonen, H. T., Foster, D. H., Wood, J. R., Wagemans, J. and Van Gool, L. (1996). Qualitative cues in the discrimination of affine-transformed minimal patterns, Perception 25, 195-206.

Lawson, R. (1999). Achieving visual object constancy across plane rotation and depth rotation, Acta Psychologica 102, 221-245.

Mardia, K. V. (1972). Statistics of Directional Data. Academic Press, New York, NY.

Mundy, J. L. and Zisserman, A. (Eds). (1992). Geometric Invariance in Computer Vision. MIT Press, Cambridge, MA.

Niall, K. (1997). Estimates of shape by eye, or, The little invariant that could, Acta Psychologica 100, $291-320$.

Niall, K. (2000). Some plane truths about pictures: notes on Wagemans, Lamote and Van Gool (1997), Spatial Vision 13, 1-24.

Niall, K. and Macnamara, J. (1989). Projective invariance and visual shape constancy, Acta Psychologica 72, 65-79.

Niall, K. and Macnamara, J. (1990). Projective invariance and picture perception, Perception 19, 637-660.

Phillips, F., Todd, J. T., Koenderink, J. J. and Kappers, A. M. L. (1997). Perceptual localization of surface slant, J. Exper. Psychol.: Human Perception and Performance 23, 1481-1492.

Pizlo, Z. (1994). A theory of shape constancy based on perspective invariants, Vision Research 34, $1637-1658$.

Simpson, W. A. (1983). The cross ratio and the perception of motion and structure, in: Motion: Representation and Perception, Badler, N. I. and Tsotsos, J. K. (Eds), pp. 248-252. North-Holland, New York.

te Pas, S. F., Kappers, A. M. L. and Koenderink, J. J. (1998). Locating the singular point in first-order optical flow fields, J. Exper. Psychol.: Human Perception and Performance 24, 1415-1430. 
Ullman, S. (1989). Aligning pictorial descriptions: An approach to object recognition, Cognition 32, $193-254$.

Van Gool, L., Moons, T., Pauwels, E. and Wagemans, J. (1994a). Invariance from the Euclidean geometer's perspective, Perception 23, 547-561.

Van Gool, L., Moons, T. and Proesmans, M. (1994b). Groups, fixed sets, symmetries, and invariants: Part I. Fixed points, fixed lines, lines of fixed points, and pencils of fixed lines, Tech. Rep. 9426, University of Leuven, Department of Electrical Engineering, Leuven, Belgium.

Van Gool, L., Proesmans, M. and Moons, T. (1995). Groups for grouping, in: Applications of Digital Image Processing XVIII (SPIE Proceedings Volume 2564, pp. 402-413), Tescher, A. G. (Ed.). SPIE, San Diego, CA.

Wagemans, J., Lamote, C. and Van Gool, L. (1997). Shape equivalence under perspective and projective transformations, Psychonomic Bulletin and Review 4, 248-253.

Wagemans, J., Van Gool, L. and Lamote, C. (1996). The visual system's measurements of invariants need not itself be invariant, Psychological Science 7, 232-236.

Wagemans, J., Van Gool, L., Lamote, C. and Foster, D. H. (2000). Minimal information to determine affine shape equivalence, J. Exper. Psychol.: Human Perception and Performance 26, 443-468.

\section{APPENDIX A}

Covariance ellipses that were centered on the 2D median of the estimations were calculated in every condition for every subject. Here we explain how we did the calculations.

The 2D median was calculated as follows. First, the one-dimensional (1D) medians along the 0,60 and 120 degrees direction (relative to the horizontal) were calculated. The 2D median was taken to be the average of the three 1D medians. A graphical presentation of these calculations is shown in Fig. A1. In order to calculate the 1D medians, the cloud of dots was projected onto each of the median

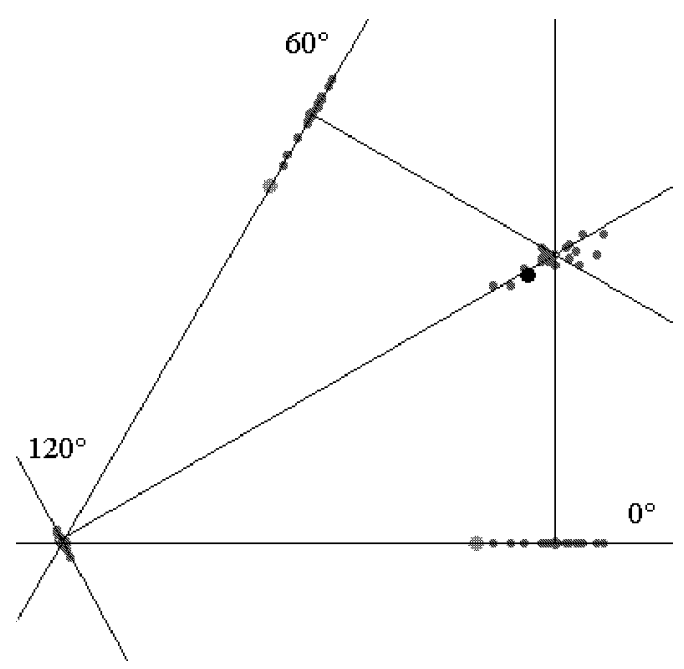

Figure A1. The 2D median was calculated by first taking the $1 \mathrm{D}$ median along the 0,60 and 120 degrees condition and then taking the average. 
axes $(0,60$ and 120 degrees). On each axis, the 1D median of these projections was calculated. Then three axes were construed, perpendicular to each of the corresponding 1D median axes. Finally, the crossing between the three axes was calculated. This was the 2D median (see Note 5).

To obtain the covariance ellipse, we first calculated the covariance matrix of the $x$ - and $y$-coordinates of the estimations in each condition separately. The lengths of the axes of the covariance ellipse of a certain condition were the first and second eigenvalues of the covariance matrix of that condition. The two (orthogonal) eigenvectors of the covariance matrix define the orientation of the ellipse. The covariance ellipse can be interpreted as the contour that delineates one standard deviation of the estimations along all possible directions.

\section{APPENDIX B}

As is shown in Fig. 7, axial data can be summarized by the length of the resultant vector, $\bar{R}$, and its orientation, $\bar{X}_{0}$. These statistics can be used to check whether there is a preferred orientation in a sample of orientations and if so, whether this orientation is significantly deviating from some theoretical value.

The value of $\bar{R}$ ranges from zero (uniformity) to one (identical orientations). Since the distribution of $\bar{R}$ is known, it is possible to calculate a critical $\bar{R}$-value for a fixed significance level, to test whether the $\bar{R}$-value of a given data set deviates from zero. If a preferred orientation exists in the data, we can check whether the hypothesis that this direction is equal to a given theoretical orientation, $\mu_{0}$, can be rejected. This can be done by setting up a confidence interval about the mean orientation, $\bar{X}_{0}$ :

$$
\left[\overline{X_{0}}-\delta, \overline{X_{0}}+\delta\right] \text {. }
$$

Given $n$, the number of data points and $\bar{R}$, the resultant length of the data points, it is possible to find the value of $\delta$ for a fixed significance level, $\alpha$. We now have a $(1-\alpha)$ confidence interval. If $\mu_{0}$ is located in this interval, the null hypothesis that the observed mean orientation, $\bar{X}_{0}$, is equal to the theoretical orientation, $\mu_{0}$, cannot be rejected. Consequently, these two orientations are statistically identical.

We applied these tests to the orientations of the covariance ellipses we obtained from our data. The critical $\bar{R}$-values for the uniformity test and the cut-off values of the confidence intervals about the mean orientation are reported in Table B1. See also Fig. 8 for a visual representation of the results. 
Table B1.

Results of the analysis of the orientation of the covariance ellipses

\begin{tabular}{|c|c|c|c|c|c|c|c|c|}
\hline & Affine $X$ & Affine $Y$ & $\bar{R}$ & $\bar{X}_{0}(\operatorname{deg})$ & $\bar{X}_{0}-\delta_{0.05}$ & $\bar{X}_{0}+\delta_{0.05}$ & $\mathrm{~S} / \mathrm{C}$ & $\chi^{2}$ \\
\hline 0 & 25 & 25 & 0.59 & 28.5 & & & & NS/NS \\
\hline 0 & 25 & 75 & $\underline{0.69}$ & 114 & 96 & 132 & $\underline{C} \& S$ & $\mathrm{NS} / \mathrm{S}$ \\
\hline 0 & 25 & 125 & $\overline{0.22}$ & 89.5 & & & & NS/NS \\
\hline 0 & 75 & 25 & 0.48 & 118 & & & & NS/NS \\
\hline 0 & 75 & 75 & 0.51 & 142 & & & & NS/NS \\
\hline 0 & 75 & 125 & 0.15 & 131.5 & & & & NS/NS \\
\hline 0 & 125 & 25 & 0.4 & 99 & & & & NS/NS \\
\hline 0 & 125 & 75 & 0.19 & 154.5 & & & & NS/NS \\
\hline 0 & 125 & 125 & 0.14 & 161 & & & & NS/NS \\
\hline 55 & 25 & 25 & 0.94 & 34 & 24.5 & 43.5 & $S$ & $\mathrm{~S} / \mathrm{S}$ \\
\hline 55 & 25 & 75 & 0.89 & 118.5 & 108 & 129 & $C$ & $\mathrm{~S} / \mathrm{S}$ \\
\hline 55 & 25 & 125 & 0.66 & 30 & & & & NS/NS \\
\hline 55 & 75 & 25 & 0.81 & 130 & 116.5 & 143.5 & $C$ & $\mathrm{~S} / \mathrm{S}$ \\
\hline 55 & 75 & 75 & 0.85 & 31.5 & 19 & 44 & $S$ & $\mathrm{~S} / \mathrm{S}$ \\
\hline 55 & 75 & 125 & 0.26 & 39.5 & & & & NS/NS \\
\hline 55 & 125 & 25 & $\underline{0.84}$ & 31.5 & 17 & 46 & $C \& \underline{S}$ & $\mathrm{NS} / \mathrm{S}$ \\
\hline 55 & 125 & 75 & $\overline{0.88}$ & 154 & 138 & 170 & $C \& \bar{S}$ & $\mathrm{NS} / \mathrm{S}$ \\
\hline 55 & 125 & 125 & $\overline{0.72}$ & 30.5 & 12 & 49 & $\underline{S}$ & $\mathrm{~S} / \mathrm{NS}$ \\
\hline 71 & 25 & 25 & $\overline{0.65}$ & 35.5 & 15.5 & 55.5 & $\underline{\bar{S}}$ & $\mathrm{~S} / \mathrm{NS}$ \\
\hline 71 & 25 & 75 & $\overline{0.77}$ & 114 & 99.5 & 128.5 & $\bar{C}$ & $\mathrm{~S} / \mathrm{S}$ \\
\hline 71 & 25 & 125 & 0.63 & 41.5 & & & & NS/NS \\
\hline 71 & 75 & 25 & 0.82 & 133 & 118.5 & 147.5 & $C$ & $\mathrm{~S} / \mathrm{S}$ \\
\hline 71 & 75 & 75 & 0.92 & 27 & 17.5 & 36.5 & $S$ & $\mathrm{~S} / \mathrm{S}$ \\
\hline 71 & 75 & 125 & 0.78 & 53.5 & 39 & 68 & $C \& S$ & $\mathrm{NS} / \mathrm{S}$ \\
\hline 71 & 125 & 25 & 0.47 & 24.5 & & & & NS/NS \\
\hline 71 & 125 & 75 & 0.67 & 39 & & & & NS/NS \\
\hline 71 & 125 & 125 & 0.97 & 26 & 19.5 & 32.5 & $S$ & $\mathrm{~S} / \mathrm{S}$ \\
\hline
\end{tabular}

(1) The first three columns contain the slant, the affine $X$-coordinate of the fourth dot to be estimated and its affine $Y$-coordinate, respectively. Conditions that are expected to yield significant orientations and where one of the axes is expected to lie in the $95 \%$ confidence interval about the mean orientation are in boldface.

(2) The italicized cells in the column containing the $\bar{R}$-values are significant at the $0.05 / 27$ level ( \pm 0.002$)$. Underlined $\bar{R}$-values refer to marginally significant values ( $p=0.01)$.

(3) Only if the $\bar{R}$-values were (marginally) significant, we also tested whether the symmetry or the collinearity-axis was in the $95 \%$ it is of no use to test for a specific orientation, if the orientations are uniformly divided. The level of $p$ was set to 0.05 . Columns 5, 6 and 7 contain the mean orientation of the covariance ellipses in that condition, the left cut-off value of the $95 \%$ confidence interval about the mean orientation and the right cut-off value. In the column with the $\mathrm{S} / \mathrm{C}$ heading, it is indicated which axis is expected to be in the confidence interval. S refers to the symmetry axis at $30 \mathrm{deg}$ and $\mathrm{C}$ to the collinearity axis at $120 \mathrm{deg}$. For the conditions in which the fourth point was off the reference axes, we did not know which axis to expect. Consequently, both of the axes are mentioned. If an axis is in the confidence interval, the cell is italicized if the corresponding $\bar{R}$-value was significant. If the $\bar{R}$-value was marginally significant, axes that are in the confidence interval are underlined.

(4) The last column contains the labels of the categorization of the conditions for $\chi^{2}$ test. See Table 1 and the last paragraph of the Results section in the text. 
Copyright of Spatial Vision is the property of VSP International Science Publishers and its content may not be copied or emailed to multiple sites or posted to a listserv without the copyright holder's express written permission. However, users may print, download, or email articles for individual use. 